\title{
A novel steady-state productivity equation for horizontal wells in bottom water drive gas reservoirs
}

\author{
Zhang Liehui ${ }^{1 *}$, Zhao Yulong ${ }^{1}$ and Liu Zhibin ${ }^{2}$ \\ ${ }^{1}$ State Key Laboratory of Oil and Gas Reservoir Geology and Exploitation, Southwest Petroleum University, Chengdu, \\ Sichuan 610500, China \\ ${ }^{2}$ School of Science, Southwest Petroleum University, Chengdu, Sichuan 610500, China
}

(C) China University of Petroleum (Beijing) and Springer-Verlag Berlin Heidelberg 2011

\begin{abstract}
It is known that there is a discrepancy between field data and the results predicted from the previous equations derived by simplifying three-dimensional (3-D) flow into two-dimensions (2-D). This paper presents a new steady-state productivity equation for horizontal wells in bottom water drive gas reservoirs. Firstly, the fundamental solution to the 3-D steady-state Laplace equation is derived with the philosophy of source and the Green function for a horizontal well located at the center of the laterally infinite gas reservoir. Then, using the fundamental solution and the Simpson integral formula, the average pseudo-pressure equation and the steady-state productivity equation are achieved for the horizontal section. Two case-studies are given in the paper, the results calculated from the newly-derived formula are very close to the numerical simulation performed with the Canadian software CMG and the real production data, indicating that the new formula can be used to predict the steady-state productivity of such horizontal gas wells.
\end{abstract}

Key words: Horizontal well, point-source function, bottom water driver gas reservoir, steady-state productivity

\section{Introduction}

Prediction of the steady-state productivity of a horizontal well is particularly important during the period of reservoir production, as more horizontal wells are being used in singlelayer reservoirs, low-permeability reservoirs, fractured reservoirs, multilayered reservoirs stratigraphically screened by an unconformity or tectonically screened by faults, and bottom water drive reservoirs. Several methods, including the analytical method, conformal transformation method, potential superposition, equivalent flow resistance method, and the point source function, were proposed to calculate the horizontal well productivity. Merkulov (1958) firstly presented an analytical equation to calculate the horizontal well productivity. Borisow (1984) reported a theoretical equation to calculate the steady-state oil production from a horizontal well; but the report did not include the derivation of the equation. Later, Giger et al $(1984 ; 1985)$ and Karcher and Giger (1986) reported reservoir engineering aspects of horizontal drilling and developed a concept of replacement ratio, $F R$, which indicates the number of vertical wells required to produce at the same rate as that of a single horizontal well. Reiss (1987) proposed a productivityindex equation for horizontal wells. Thereafter Joshi (1988)

*Corresponding author. email: zhangliehui@vip.163.com

Received August 18, 2010 derived an equation to calculate the productivity of steadystate horizontal wells using potential-fluid theory. Babu and Odeh $(1989 ; 1991)$ reduced a complex equation to an easyto-use equation for calculating the productivity of horizontal wells, requiring that the drainage volume be approximately box-shaped, and all the boundaries of the drainage volume be closed. Renard and Dupuy (1991) took the skin factor into consideration and derived the flow efficiency equation for horizontal wells in anisotropic reservoirs. Larsen (1996) studied the pseudosteady-state productivity index and skin factor for multilateral, branched and extended wells. Helmy and Wattenbarger (1998) presented a simple equation to calculate the productivity of a horizontal well when producing at a constant bottomhole flowing pressure or at a constant rate in bounded reservoirs. Chen et al (2000) developed a deliverability model by calculating the productivity and pressure of each branch, and combining them to predict the performance of multilateral wells. Through considering the non-Darcy flow effect and mechanical skin effect in Babu and Odeh's model, Billiter et al (2001) applied the analytical methods and Monte Carlo simulation to yield the equation of dimensionless inflow-performance-relationship (IPR) curves. Anklam and Wiggins (2005) obtained the wellbore pressure along the well and the steady-state productivity with the consideration of the mechanical properties of fluid flow in the wellbore. Lu et al $(1993 ; 2001 ; 2002 ; 2003)$ achieved the productivity equations for horizontal wells under different boundary conditions. These equations are based on the point 
source function.

Most of the results above are mainly for oil reservoirs, and there are only a few studies reported on the productivity of horizontal wells in gas reservoirs, especially for the bottomwater-drive gas reservoirs. In this paper, we employed a similar method to that described by $\mathrm{Lu}(2001 ; 2002)$ and derived the steady-state productivity formula for a horizontal well located at the center of a laterally infinite, bottom waterdrive gas reservoir.

\section{The horizontal well model}

\subsection{Assumptions}

Fig. 1 presents the configuration of a horizontal well drilled in a bottom water drive gas reservoir, which is assumed to be laterally infinite. To make the problem more tractable, the following assumptions are made: 1) The porous medium is homogeneous and anisotropic with constant permeability $k_{\mathrm{x}}, k_{\mathrm{y}}$, and $k_{\mathrm{z}}$ and porosity of . 2) The reservoir is horizontal and of uniform thickness of $H$. 3) The initial reservoir pressure $p_{\mathrm{i}}$ is constant; the upper boundary is closed to flow while the lower boundary is at constant-pressure. 4) The single-phase fluid is compressible and flows from the reservoir to the well at a constant rate, $Q_{\mathrm{sc}}$.

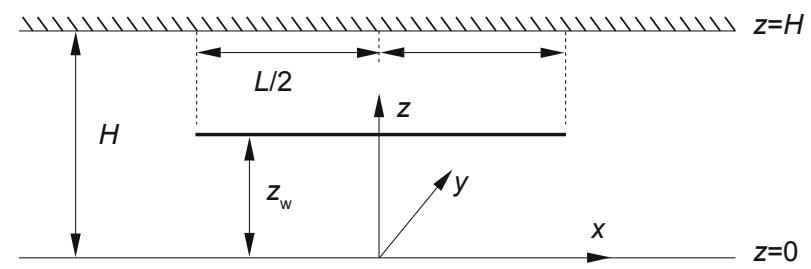

Fig. 1 Schematic of a horizontal well in a bottom-water-drive gas reservoir

\subsection{Steady-state equation}

According to the theory of source and the Green function (Carslaw and Jaeger, 1959; Gringarten and Ramey, 1973), and substituting the quality of the point source $\rho_{\mathrm{sc}} q_{\mathrm{sc}}$ at the point $\left(x_{\mathrm{o}}, 0, z_{\mathrm{w}}\right)$ into the flow equation, we have

$$
\begin{aligned}
& -\left[\frac{\partial\left(\rho v_{x}\right)}{\partial x}+\frac{\partial\left(\rho v_{y}\right)}{\partial y}+\frac{\partial\left(\rho v_{z}\right)}{\partial z}\right] \\
& =\rho_{\mathrm{sc}} q_{\mathrm{sc}} \delta\left(x-x_{\mathrm{o}}\right) \delta(y) \delta\left(z-z_{\mathrm{w}}\right)
\end{aligned}
$$

where $(x, y, z)$ is the observation point, $\left(x_{0}, 0, z_{\mathrm{w}}\right)$ is a point in the source point region, and $x, y, z$ are the coordinates, $\mathrm{m} ; q_{\mathrm{sc}}$ is the point convergence intensity (flow rate), $\mathrm{m}^{3} / \mathrm{s}, \rho$ and $\rho_{\text {sc }}$ are the fluid density under the reservoir and standard conditions, respectively, $\mathrm{g} / \mathrm{m}^{3}, v_{x}, v_{y}, v_{z}$ are the fluid velocity in the $x, y, z$ directions respectively, $\mathrm{m} / \mathrm{s} ; z_{\mathrm{w}}$ is the distance between the horizontal well and the bottom of the reservoir; $\delta\left(x-x_{\mathrm{o}}\right), \delta(y), \delta\left(z-z_{\mathrm{w}}\right)$ are Dirac functions.

Darcy equation:

$$
v_{x}=-\frac{k_{x}}{\mu} \frac{\partial p}{\partial x}, \quad v_{y}=-\frac{k_{y}}{\mu} \frac{\partial p}{\partial y}, \quad v_{z}=-\frac{k_{z}}{\mu} \frac{\partial p}{\partial z}
$$

State equation:

$$
p M=\rho Z R T
$$

Substituting Eqs. (2) and (3) into Eq. (1) gives:

$$
\begin{aligned}
& k_{x} \frac{\partial\left(\frac{p}{\mu Z} \frac{\partial p}{\partial x}\right)}{\partial x}+k_{y} \frac{\partial\left(\frac{p}{Z \mu} \frac{\partial p}{\partial y}\right)}{\partial y}+k_{z} \frac{\partial\left(\frac{p}{Z \mu} \frac{\partial p}{\partial z}\right)}{\partial z} \\
& =\frac{T}{T_{\mathrm{sc}}} p_{\mathrm{sc}} q_{\mathrm{sc}} \delta\left(x-x_{\mathrm{o}}\right) \delta(y) \delta\left(z-z_{\mathrm{w}}\right)
\end{aligned}
$$

where $p$ is the reservoir pressure, $\mathrm{MPa} ; T$ is the reservoir temperature, $\mathrm{K} ; p_{\mathrm{sc}}$ is the standard pressure, $p_{\mathrm{sc}}=0.1 \mathrm{MPa} ; T_{\mathrm{sc}}$ is the standard temperature, $T_{\mathrm{sc}}=273 \mathrm{~K} ; k$ is the permeability, $\mathrm{D} ; \mu$ is the gas viscosity, $\mathrm{mPa} \cdot \mathrm{s} ; Z$ is the compressibility factor for gas, fraction; $R$ is the universal gas constant, $R=8.314 \mathrm{~J} /$ $(\mathrm{K} \cdot \mathrm{mol}) ; M$ is the molar mass of natural gas, $\mathrm{mol} / \mathrm{kg}$.

Here, the pseudo-pressure $m(p)$ is:

$$
m(p)=2 \int_{p_{\mathrm{o}}}^{p} \frac{p}{\mu Z} \mathrm{~d} p
$$

where $p_{\mathrm{o}}$ is the reference pressure, MPa.

Introducing Eq. (5) into Eq. (4) yields:

$$
\begin{aligned}
& k_{x} \frac{\partial^{2} m}{\partial x^{2}}+k_{y} \frac{\partial^{2} m}{\partial y^{2}}+k_{z} \frac{\partial^{2} m}{\partial z^{2}} \\
& =2 \frac{T}{T_{\mathrm{sc}}} p_{\mathrm{sc}} q_{\mathrm{sc}} \delta\left(x-x_{\mathrm{o}}\right) \delta(y) \delta\left(z-z_{\mathrm{w}}\right)
\end{aligned}
$$

\subsection{Dimensionless steady-state equation}

Various dimensionless groups are defined as:

$$
\begin{aligned}
& \bar{k}=\left(k_{x} k_{y} k_{z}\right)^{1 / 3}, \quad m_{\mathrm{D}}=\frac{\pi \bar{k} L T_{\mathrm{sc}}}{q_{\mathrm{sc}} p_{\mathrm{sc}} T}\left(m_{\mathrm{i}}-m\right) \\
& x_{\mathrm{D}}=\frac{x}{L}\left(\frac{\bar{k}}{k_{x}}\right)^{1 / 2}, \quad y_{\mathrm{D}}=\frac{y}{L}\left(\frac{\bar{k}}{k_{y}}\right)^{1 / 2}, \quad z_{\mathrm{D}}=\frac{z}{L}\left(\frac{\bar{k}}{k_{z}}\right)^{1 / 2} \\
& H_{\mathrm{D}}=\frac{H}{L}\left(\frac{\bar{k}}{k_{z}}\right)^{1 / 2}, \quad L_{\mathrm{D}}=\left(\frac{\bar{k}}{k_{y}}\right)^{1 / 2}, \quad z_{\mathrm{wD}}=\frac{z_{\mathrm{w}}}{L}\left(\frac{\bar{k}}{k_{z}}\right)^{1 / 2} \\
& \beta=\sqrt{\frac{k_{\mathrm{h}}}{k_{\mathrm{v}}}}, \quad R_{\mathrm{wD}}=\left(\beta^{1 / 2}+\beta^{-1 / 2}\right) \frac{R_{\mathrm{w}}}{2 L}
\end{aligned}
$$

Then, we have

$$
\begin{aligned}
& k_{x} \frac{\partial^{2} m}{\partial x^{2}}=-k_{x} \frac{\partial}{\partial x}\left[\frac{q_{\mathrm{sc}} p_{\mathrm{sc}} T}{\pi \bar{k} L^{2} T_{\mathrm{sc}}}\left(\frac{\bar{k}}{k_{x}}\right)^{1 / 2} \frac{\partial m_{\mathrm{D}}}{\partial x_{\mathrm{D}}}\right] \\
& =-\frac{q_{\mathrm{sc}} p_{\mathrm{sc}} T}{\pi L^{3} T_{\mathrm{sc}}} \frac{\partial^{2} m_{\mathrm{D}}}{\partial x_{\mathrm{D}}{ }^{2}}
\end{aligned}
$$

Similarly

$$
\begin{aligned}
& k_{y} \frac{\partial^{2} m}{\partial y^{2}}=-\frac{q_{\mathrm{sc}} p_{\mathrm{sc}} T}{\pi L^{3} T_{\mathrm{sc}}} \frac{\partial^{2} m_{\mathrm{D}}}{\partial y_{\mathrm{D}}{ }^{2}} \\
& k_{z} \frac{\partial^{2} m}{\partial z^{2}}=-\frac{q_{\mathrm{sc}} p_{\mathrm{sc}} T}{\pi L^{3} T_{\mathrm{sc}}} \frac{\partial^{2} m_{\mathrm{D}}}{\partial z_{\mathrm{D}}{ }^{2}}
\end{aligned}
$$


where $m_{\mathrm{i}}$ is the pseudo-pressure under the reservoir pressure; $H$ is the effect reservoir thickness, m; $L$ is the well length, m; $r_{\mathrm{w}}$ is the wellbore radius, $\mathrm{m} ; \bar{k}$ is the effective permeability.

Because $\delta(c x)=(x) / c$ (where $c$ is a positive constant (Zwillinger, 1996)) we have

$$
\begin{aligned}
& 2 \frac{T}{T_{\mathrm{sc}}} p_{\mathrm{sc}} q_{\mathrm{sc}} \delta\left(x-x_{\mathrm{o}}\right) \delta(y) \delta\left(z-z_{\mathrm{w}}\right) \\
& =\frac{2 T}{T_{\mathrm{sc}}} p_{\mathrm{sc}} q_{\mathrm{sc}} \frac{\bar{k}^{3 / 2}}{L^{3}\left(k_{x} k_{y} k_{\mathrm{z}}\right)^{1 / 2}} \delta\left(x_{\mathrm{D}}-x_{\mathrm{oD}}\right) \delta\left(y_{\mathrm{D}}\right) \delta\left(z_{\mathrm{D}}-z_{\mathrm{wD}}\right)
\end{aligned}
$$

where $x_{\mathrm{oD}}$ and $z_{\mathrm{oD}}$ are the dimensionless coordinates of the point source in the $x$ and $z$ directions, respectively.

Hence, combining Eqs. (7)-(9) with Eq. (6) yields:

$$
\begin{aligned}
& \frac{q_{\mathrm{sc}} p_{\mathrm{sc}} T}{\pi L^{3} T_{\mathrm{sc}}}\left(\frac{\partial^{2} m_{\mathrm{D}}}{\partial x_{\mathrm{D}}{ }^{2}}+\frac{\partial^{2} m_{\mathrm{D}}}{\partial y_{\mathrm{D}}{ }^{2}}+\frac{\partial^{2} m_{\mathrm{D}}}{\partial z_{\mathrm{D}}{ }^{2}}\right) \\
& =-\frac{2 T}{T_{\mathrm{sc}} L^{3}} p_{\mathrm{sc}} q_{\mathrm{sc}} \frac{(\bar{k})^{3 / 2}}{\left(k_{x} k_{y} k_{\mathrm{z}}\right)^{1 / 2}} \delta\left(x_{\mathrm{D}}-x_{0 \mathrm{D}}\right) \delta\left(y_{\mathrm{D}}\right) \delta\left(z_{\mathrm{D}}-z_{\mathrm{wD}}\right)
\end{aligned}
$$

Eq. (11) can be simplified as:

$$
\begin{aligned}
& \frac{\partial^{2} m_{\mathrm{D}}}{\partial x_{\mathrm{D}}{ }^{2}}+\frac{\partial^{2} m_{\mathrm{D}}}{\partial y_{\mathrm{D}}{ }^{2}}+\frac{\partial^{2} m_{\mathrm{D}}}{\partial z_{\mathrm{D}}{ }^{2}} \\
& =-2 \pi \delta\left(x_{\mathrm{D}}-x_{\mathrm{oD}}\right) \delta\left(y_{\mathrm{D}}\right) \delta\left(z_{\mathrm{D}}-z_{\mathrm{wD}}\right)
\end{aligned}
$$

Here, it has to be clarified if the point convergence density (flow rate) of the point $\left(x_{\mathrm{o}}, 0, z_{\mathrm{w}}\right)$ is $q_{\mathrm{sc}}$, then the total productivity of the horizontal well is ( $\mathrm{Lu}, 2001)$ :

$$
Q_{\mathrm{sc}}=86.4 q_{\mathrm{sc}} L_{\mathrm{D}}
$$

where $Q_{\mathrm{sc}}$ is the total productivity of the horizontal well, $10^{4} \mathrm{~m}^{3} /$ day

\subsection{Point source solution}

The horizontal well produces from a bottom-water-drive gas reservoir. The upper and lower boundary conditions can be expressed in the form of pseudo-pressure:

$$
\left.m_{\mathrm{D}}\right|_{z_{\mathrm{D}}=0}=0,\left.\quad \frac{\partial m_{\mathrm{D}}}{\partial z_{\mathrm{D}}}\right|_{z_{\mathrm{D}}=H_{\mathrm{D}}}=0
$$

From Eq. (14), the characteristic function of the differential equation with these boundaries is $z\left(\beta_{n}, z_{\mathrm{D}}\right)=\sin \frac{(2 n-1) \pi z_{\mathrm{D}}}{2 H_{\mathrm{D}}}$, and its characteristic value is $\beta_{n}=\frac{(2 n-1) \pi}{2 H_{\mathrm{D}}},(n=1,2,3, \cdots,+\infty)$.

Applying the following finite Fourier sine transform to $z_{\mathrm{D}}$ (Kong, 1999), given

$$
U\left(x_{\mathrm{D}}, y_{\mathrm{D}}, \beta_{n}\right)=\int_{0}^{H_{\mathrm{D}}} m_{\mathrm{D}}\left(x_{\mathrm{D}}, y_{\mathrm{D}}, z_{\mathrm{D}}\right) \sin \frac{(2 n-1) \pi z_{\mathrm{D}}}{2 H_{\mathrm{D}}} \mathrm{d} z_{\mathrm{D}}
$$

So from Eq. (12), we have

$$
\begin{aligned}
& -\int_{0}^{H_{\mathrm{D}}} 2 \pi \delta\left(x_{\mathrm{D}}-x_{\mathrm{OD}}\right) \delta\left(y_{\mathrm{D}}\right) \delta\left(z_{\mathrm{D}}-z_{\mathrm{wD}}\right) \sin \frac{(2 n-1) \pi z_{\mathrm{D}}}{2 H_{\mathrm{D}}} \mathrm{d} z_{\mathrm{D}} \\
& =\int_{0}^{H_{\mathrm{D}}}\left(\frac{\partial^{2} m_{\mathrm{D}}}{\partial x_{\mathrm{D}}{ }^{2}}+\frac{\partial^{2} m_{\mathrm{D}}}{\partial y_{\mathrm{D}}{ }^{2}}+\frac{\partial^{2} m_{\mathrm{D}}}{\partial z_{\mathrm{D}}{ }^{2}}\right) \sin \frac{(2 n-1) \pi z_{\mathrm{D}}}{2 H_{\mathrm{D}}} \mathrm{d} z_{\mathrm{D}} \\
& =\int_{0}^{H_{\mathrm{D}}}\left(\frac{\partial^{2} m_{\mathrm{D}}}{\partial x_{\mathrm{D}}{ }^{2}}+\frac{\partial^{2} m_{\mathrm{D}}}{\partial y_{\mathrm{D}}{ }^{2}}\right) \sin \frac{(2 n-1) \pi z_{\mathrm{D}}}{2 H_{\mathrm{D}}} \mathrm{d} z_{\mathrm{D}} \\
& +\int_{0}^{H_{\mathrm{D}}} \frac{\partial^{2} m_{\mathrm{D}}}{\partial z_{\mathrm{D}}{ }^{2}} \sin \frac{(2 n-1) \pi z_{\mathrm{D}}}{2 H_{\mathrm{D}}} \mathrm{d} z_{\mathrm{D}}
\end{aligned}
$$

Combining Eq. (15) with Eq. (16) yields:

$$
\frac{\partial^{2} U}{\partial x_{\mathrm{D}}{ }^{2}}+\frac{\partial^{2} U}{\partial y_{\mathrm{D}}{ }^{2}}-\beta_{n}{ }^{2} U=-2 \pi \delta\left(x_{\mathrm{D}}-x_{\mathrm{oD}}\right) \delta\left(y_{\mathrm{D}}\right) \sin \left(\beta_{n} z_{\mathrm{wD}}\right)
$$

From the fundamental solution to the modified Helmholtz function (Zwillinger, 1996), we obtain the fundamental solution to Eq. (17):

$$
U\left(x_{\mathrm{D}}, y_{\mathrm{D}}, \beta_{n}\right)=\sin \left(\beta_{n} z_{\mathrm{wD}}\right) K_{0}\left(R_{\mathrm{D}} \beta_{n}\right)
$$

with

$$
R_{\mathrm{D}}=\sqrt{\left(x_{\mathrm{D}}-x_{\mathrm{oD}}\right)^{2}+y_{\mathrm{D}}^{2}}
$$

According to the inverse Fourier sine transform, there is

$$
\begin{aligned}
& m_{\mathrm{D}}\left(x_{\mathrm{D}}, y_{\mathrm{D}}, z_{\mathrm{D}}\right) \\
& =\sum_{n=1}^{\infty} \frac{\sin \frac{(2 n-1) \pi z_{\mathrm{D}}}{2 H_{\mathrm{D}}}}{\int_{0} \sin ^{2} \frac{(2 n-1) \pi z_{\mathrm{D}}}{2 H_{\mathrm{D}}} \mathrm{d} z_{\mathrm{D}}} U\left(x_{\mathrm{D}}, y_{\mathrm{D}}, \beta_{n}\right) \\
& =\frac{2}{H_{\mathrm{D}}} \sum_{n=1}^{\infty} \sin \left(\beta_{n} z_{\mathrm{D}}\right) \sin \left(\beta_{n} z_{\mathrm{wD}}\right) K_{0}\left(R_{\mathrm{D}} \beta_{n}\right)
\end{aligned}
$$

The dimensionless pseudo-pressure of the point source at the point $\left(x_{\mathrm{oD}}, 0, z_{\mathrm{wD}}\right)$ can be achieved by simplifying Eq. (19):

$$
m_{\mathrm{D}}\left(x_{\mathrm{D}}, y_{\mathrm{D}}, z_{\mathrm{D}}\right)=\frac{2}{H_{\mathrm{D}}} \sum_{n=1}^{\infty} \sin \beta_{n} z_{\mathrm{D}} \sin \beta_{n} z_{\mathrm{wD}} K_{0}\left(R_{\mathrm{D}} \beta_{n}\right)
$$

\section{Steady-state productivity equation}

\subsection{Dimensionless wellbore pseudo-pressure}

The dimensionless wellbore radius is:

$$
R_{\mathrm{wD}}=\sqrt{\left(x_{\mathrm{wD}}-x_{0 \mathrm{D}}\right)^{2}+y_{\mathrm{wD}}^{2}}
$$

where $x_{\mathrm{wD}}$ and $y_{\mathrm{wD}}$ are the dimensionless coordinates in the $x$ and $y$ directions at the wellbore, respectively.

Note that $L>>R_{\mathrm{wD}}$, then $y_{\mathrm{wD}} \approx 0$, so

$$
R_{\mathrm{wD}} \approx\left|x_{\mathrm{wD}}-x_{\mathrm{oD}}\right|
$$

If the point $\left(x_{\mathrm{w}}, 0, z_{\mathrm{w}}\right)$ is on the horizontal line source of 
the wellbore and the boundary conditions satisfy Eq. (14), then, in Eq. (20) we can assume

$$
z_{\mathrm{D}}=z_{\mathrm{wD}}+R_{\mathrm{wD}}
$$

Let $x_{\mathrm{oD}}$ be the element of integration along the horizontal section in the interval $\left(-L_{\mathrm{D}} / 2, L_{\mathrm{D}} / 2\right)$, the dimensionless pseudo-pressure of the horizontal well at the point $\left(x_{\mathrm{wD}}, 0\right.$, $z_{\mathrm{wD}}$ ) can be achieved by the superposition of the potential field:

$$
m_{\mathrm{wD}}=\int_{-L_{\mathrm{D}} / 2}^{L_{\mathrm{D}} / 2} m_{\mathrm{D}} \mathrm{d} x_{\mathrm{oD}}
$$

Combining Eq. (20) with Eq. (23) gives:

$$
m_{\mathrm{wD}}=\int_{-L_{\mathrm{D}} / 2}^{L_{\mathrm{D}} / 2} \frac{2}{H_{\mathrm{D}}} \sum_{n=1}^{\infty} \sin \left(\beta_{n} z_{\mathrm{D}}\right) \sin \left(\beta_{n} z_{\mathrm{wD}}\right) K_{0}\left(R_{\mathrm{wD}} \beta_{n}\right) \mathrm{d} x_{\mathrm{oD}}
$$

Combining Eqs. (21) and (22) with Eq. (24) gives:

$$
\left.m_{\mathrm{wD}}=\int_{-L_{\mathrm{D}} / 2}^{L_{\mathrm{D}} / 2} \frac{2}{H_{\mathrm{D}}} \sum_{n=1}^{\infty} \sin \left(\beta_{n} z_{\mathrm{wD}}\right) K_{0}\left(\left|x_{\mathrm{wD}}-x_{\mathrm{oD}}\right| \beta_{n}\right)^{\mathrm{d}}\left(z_{\mathrm{wD}}+R_{\mathrm{wD}}\right)\right] \times \quad
$$

1) If the target points are the two end points of the horizontal well, $x_{\mathrm{wD}}= \pm L_{\mathrm{D}} / 2$, then we have

$$
m_{\mathrm{wD}}^{\mathrm{E}}=\int_{-L_{\mathrm{D}} / 2}^{L_{\mathrm{D}} / 2} \frac{2}{H_{\mathrm{D}}} \sum_{n=1}^{\infty} \sin \left(\beta_{n} z_{\mathrm{wD}}\right) K_{0}\left(\left|\frac{L_{\mathrm{D}}}{2}-x_{\mathrm{oD}}\right| \beta_{n}\right)^{\mathrm{d} x_{\mathrm{oD}}}
$$

The right side of Eq. (26) can be written as follows:

$$
\begin{aligned}
& \int_{-L_{\mathrm{D}} / 2}^{L_{\mathrm{D}} / 2} \frac{2}{H_{\mathrm{D}}} \sum_{n=1}^{\infty} \sin \left(\beta_{n} z_{\mathrm{wD}}\right) K_{0}\left(\left|\frac{L_{\mathrm{D}}}{2}-x_{\mathrm{oD}}\right| \beta_{n}\right)^{\mathrm{d} x_{\mathrm{oD}}} \\
& \left.=\frac{2}{H_{\mathrm{D}}} \sum_{n=1}^{\infty} \int_{L_{\mathrm{D}} / 2}^{L_{\mathrm{D}} / 2} \sin \left(z_{\mathrm{wD}}+R_{n}\right)\right] \times \\
& \left.\sin \left(z_{\mathrm{wD}}+R_{\mathrm{wD}}\right)\right] \times \\
& K_{0}\left(\left|\frac{L_{\mathrm{D}}}{2}-x_{\mathrm{oD}}\right| \beta_{n}\right)^{\mathrm{d} x_{\mathrm{oD}}}
\end{aligned}
$$

Let

$$
\begin{aligned}
& A_{n}^{\mathrm{E}}=\int_{-L_{\mathrm{D}} / 2}^{L_{\mathrm{D}} / 2} K_{0}\left(\left|L_{\mathrm{D}} / 2-x_{\mathrm{oD}}\right| \beta_{n}\right) \mathrm{d} x_{\mathrm{oD}} \\
& B_{n}=\sin \left[\beta_{n}\left(z_{\mathrm{wD}}+R_{\mathrm{wD}}\right)\right] \sin \left(\beta_{n} z_{\mathrm{wD}}\right)
\end{aligned}
$$

Then

$$
\begin{aligned}
& \frac{2}{H_{\mathrm{D}}} \int_{-L_{\mathrm{D}} / 2}^{L_{\mathrm{D}} / 2} \sum_{n=1}^{\infty} \sin \left(\beta_{n} z_{\mathrm{wD}}\right) K_{0}\left(\frac{L_{\mathrm{D}}}{2}-x_{\mathrm{oD}} \mid \beta_{n}\right)^{\mathrm{d} x_{\mathrm{oD}}} \\
& =\frac{2}{H_{\mathrm{D}}} \sum_{n=1}^{\infty} A_{n}{ }^{\mathrm{E}} B_{n}
\end{aligned}
$$

Using the similar derivation of Appendix D in the literature (Lu, 2001), we obtain $A_{n}^{\mathrm{E}}=\frac{H_{\mathrm{D}}}{2 n-1}$. Then Eq. (28) can be rewritten as follows:

$$
\begin{aligned}
& \frac{2}{H_{\mathrm{D}}} \sum_{n=1}^{\infty} A_{n}{ }^{\mathrm{E}} B_{n}=\frac{2}{H_{\mathrm{D}}} \sum_{n=1}^{\infty} \frac{H_{\mathrm{D}}}{2 n-1} B_{n} \\
& =2 \sum_{n=1}^{\infty} \frac{\sin \left[\beta_{n}\left(z_{\mathrm{wD}}+R_{\mathrm{wD}}\right)\right] \sin \left(\beta_{n} z_{\mathrm{wD}}\right)}{2 n-1}
\end{aligned}
$$

Using the following expression

$$
\begin{aligned}
& \sin \left[\beta_{n}\left(z_{\mathrm{wD}}+R_{\mathrm{wD}}\right)\right] \sin \left(\beta_{n} z_{\mathrm{wD}}\right) \\
& =\frac{1}{2}\left[\cos \left(\beta_{n} R_{\mathrm{wD}}\right)-\cos \left[\beta_{n}\left(2 z_{\mathrm{wD}}+R_{\mathrm{wD}}\right)\right]\right]
\end{aligned}
$$

Eq. (29) can be simplified as:

$$
\frac{2}{H_{\mathrm{D}}} \sum_{n=1}^{\infty} A_{n}^{\mathrm{E}} B_{n}=\sum_{n=1}^{\infty} \frac{\cos \left(\beta_{n} R_{\mathrm{wD}}\right)-\cos \left[\beta_{n}\left(2 z_{\mathrm{wD}}+R_{\mathrm{wD}}\right)\right]}{2 n-1}
$$

When $x \neq 0$, we have (Gradshteyn and Ryzhik, 2007)

$$
\sum_{n=1}^{\infty} \frac{\cos [(2 n-1) x]}{2 n-1}=\frac{1}{2} \ln \left[\cot \left(\frac{x}{2}\right)\right]
$$

Because $\beta_{n}=\frac{(2 n-1) \pi}{2 H_{\mathrm{D}}}$, so Eq. (30) and Eq. (31) can be combined to give:

$$
\begin{aligned}
& \frac{2}{H_{\mathrm{D}}} \sum_{n=1}^{\infty} A_{n}{ }^{\mathrm{E}} B_{n} \\
& =\sum_{n=1}^{\infty} \frac{\cos \left(\beta_{n} R_{\mathrm{wD}}\right)}{2 n-1}-\sum_{n=1}^{\infty} \frac{\cos \left[\beta_{n}\left(2 z_{\mathrm{wD}}+R_{\mathrm{wD}}\right)\right]}{2 n-1} \\
& =\frac{1}{2}\left[\ln \cot \left(\frac{\pi R_{\mathrm{wD}}}{4 H_{\mathrm{D}}}\right)-\ln \cot \left(\frac{\pi\left(2 z_{\mathrm{wD}}+R_{\mathrm{wD}}\right)}{4 H_{\mathrm{D}}}\right)\right]
\end{aligned}
$$

Incorporating Eq. (26) into Eq. (32) yields the dimensionless pseudo-pressure at the end points of the horizontal well:

$$
m_{\mathrm{wD}}^{\mathrm{E}}=\frac{1}{2} \ln \left[\ln \cot \left(\frac{\pi R_{\mathrm{wD}}}{4 H_{\mathrm{D}}}\right)-\ln \cot \left(\frac{\pi\left(2 z_{\mathrm{wD}}+R_{\mathrm{wD}}\right)}{4 H_{\mathrm{D}}}\right)\right]
$$

Because the dimensionless distance of the horizontal well to the bottom boundary is larger than the dimensionless wellbore radius $\left(z>R_{\mathrm{wD}}\right)$, so we have $2 z_{\mathrm{wD}}+R_{\mathrm{wD}} \approx 2 z_{\mathrm{wD}}$. Eq. (33) can be simplified as:

$$
m_{\mathrm{wD}}^{\mathrm{E}}=\frac{1}{2}\left\{\ln \left[\cot \left(\frac{\pi R_{\mathrm{wD}}}{4 H_{\mathrm{D}}}\right)\right]-\ln \left[\cot \left(\frac{\pi z_{\mathrm{wD}}}{2 H_{\mathrm{D}}}\right)\right]\right\}
$$

2) If the target point is the midpoint of the horizontal well, $x_{\mathrm{oD}}=0$, we have

$$
m_{\mathrm{wD}}^{\mathrm{M}}=\int_{-L_{\mathrm{D}} / 2}^{L_{\mathrm{D}} / 2} \frac{2}{H_{\mathrm{D}}} \sum_{n=1}^{\infty} \sin \beta_{n} z_{\mathrm{D}} \sin \beta_{n} z_{\mathrm{wD}} K_{0}\left(\left|0-x_{\mathrm{oD}}\right| \beta_{n}\right) \mathrm{d} x_{\mathrm{oD}}
$$


The dimensionless pseudo-pressure at the midpoint of the horizontal well is achieved using the method similar to the two end points above:

$$
m_{\mathrm{wD}}^{\mathrm{M}}=\ln \left[\cot \left(\frac{\pi R_{\mathrm{wD}}}{4 H_{\mathrm{D}}}\right)\right]-\ln \left[\cot \left(\frac{\pi z_{\mathrm{wD}}}{2 H_{\mathrm{D}}}\right)\right]
$$

According to Simpson's quadrature rule ( $\mathrm{Lu}, 2001)$, we obtain the average dimensionless pseudo-pressure along the horizontal section:

$$
\begin{aligned}
& m_{\mathrm{wD}}^{\mathrm{A}} \approx \frac{1}{6}\left(2 m_{\mathrm{wD}}^{\mathrm{E}}+4 m_{\mathrm{wD}}^{\mathrm{M}}\right) \\
& =\frac{5}{6}\left\{\ln \left[\cot \left(\frac{\pi R_{\mathrm{wD}}}{4 H_{\mathrm{D}}}\right)\right]-\ln \left[\cot \left(\frac{\pi z_{\mathrm{wD}}}{2 H_{\mathrm{D}}}\right)\right]\right\}
\end{aligned}
$$

\subsection{Horizontal well productivity}

The expression for dimensionless pseudo-pressure gives:

$m_{\mathrm{wD}}^{\mathrm{A}}=\frac{\pi \bar{k} L T_{\mathrm{sc}}}{q_{\mathrm{sc}} p_{\mathrm{sc}} T}\left(m_{\mathrm{i}}-m_{\mathrm{w}}^{\mathrm{A}}\right)$

Combining Eq. (37) with Eq. (38), we obtain:

$$
\begin{aligned}
& m_{\mathrm{wD}}^{\mathrm{A}}=\frac{5}{6}\left\{\ln \left[\cot \left(\frac{\pi R_{\mathrm{wD}}}{4 H_{\mathrm{D}}}\right)\right]-\ln \left[\cot \left(\frac{\pi z_{\mathrm{wD}}}{2 H_{\mathrm{D}}}\right)\right]\right\} \\
& =\frac{\pi \bar{k} L T_{\mathrm{sc}}}{q_{\mathrm{sc}} p_{\mathrm{sc}} T}\left(m_{\mathrm{i}}-m_{\mathrm{w}}^{\mathrm{A}}\right)
\end{aligned}
$$

Then the productivity equation for a horizontal well in a laterally infinite gas reservoir with a bottom water drive can be obtained:

$$
q_{\mathrm{sc}}=\frac{\pi \bar{k} L T_{\mathrm{sc}}}{p_{\mathrm{sc}} T} \frac{\left(m_{\mathrm{i}}-m_{\mathrm{w}}^{A}\right)}{\frac{5}{6}\left\{\ln \left[\cot \left(\frac{\pi R_{\mathrm{wD}}}{4 H_{\mathrm{D}}}\right)\right]-\ln \left[\cot \left(\frac{\pi z_{\mathrm{wD}}}{2 H_{\mathrm{D}}}\right)\right]\right\}}
$$

Note that $-\ln \cot x=\ln \tan x$ and when $x$ is infinitely small, $\tan x \sim x$, then we have:

$$
q_{\mathrm{sc}}=\frac{\pi \bar{k} L T_{\mathrm{sc}}}{p_{\mathrm{sc}} T} \frac{\left(m_{\mathrm{i}}-m_{\mathrm{w}}^{\mathrm{A}}\right)}{\frac{5}{6}\left(\ln \frac{4 H_{\mathrm{D}}}{\pi R_{\mathrm{wD}}}+\ln \tan \frac{\pi z_{\mathrm{wD}}}{2 H_{\mathrm{D}}}\right)}
$$

According to the definition of pseudo-pressure, $m(p)=2 \int_{p_{0}}^{p} \frac{p}{\mu Z} \mathrm{~d} p$, we have

$$
m_{\mathrm{i}}=2 \int_{p_{\mathrm{o}}}^{p_{\mathrm{i}}} \frac{p}{\mu Z} \mathrm{~d} p, \quad m_{\mathrm{w}}^{\mathrm{A}}=2 \int_{p_{\mathrm{o}}}^{p_{\mathrm{w}}^{\mathrm{A}}} \frac{p}{\mu Z} \mathrm{~d} p
$$

1) When $p>21 \mathrm{MPa}, \frac{p}{\mu Z}=$ Const, so

$$
\begin{aligned}
& m(p)=2 \int_{p_{\mathrm{o}}}^{p} \frac{p}{\mu Z} \mathrm{~d} p=2 \frac{\bar{p}}{\bar{\mu} \bar{Z}}\left(p-p_{\mathrm{o}}\right), \text { then } \\
& m_{\mathrm{i}}-m_{\mathrm{w}}^{\mathrm{A}}=2 \int_{p_{\mathrm{o}}}^{p_{\mathrm{i}}} \frac{p}{\mu Z} \mathrm{~d} p-2 \int_{p_{\mathrm{o}}}^{p_{\mathrm{w}}^{\mathrm{A}}} \frac{p}{\mu Z} \mathrm{~d} p=\frac{2 \bar{p}}{\bar{\mu} \bar{Z}}\left(p_{\mathrm{i}}-p_{\mathrm{w}}^{\mathrm{A}}\right)
\end{aligned}
$$

2) When $13 \mathrm{MPa}<p<21 \mathrm{MPa}$, the pseudo-pressure should not be simplified.

3) When $\mathrm{p}<13 \mathrm{MPa}, \mu Z=$ Const, so

$$
\begin{aligned}
& m(p)=2 \int_{p_{\mathrm{o}}}^{p} \frac{p}{\mu Z} \mathrm{~d} p=\frac{1}{\mu \bar{Z}}\left(p^{2}-p_{\mathrm{o}}{ }^{2}\right), \text { then } \\
& m_{\mathrm{i}}-m_{\mathrm{w}}^{A}=2 \int_{p_{\mathrm{o}}}^{p_{\mathrm{i}}} \frac{p}{\mu Z} \mathrm{~d} p-2 \int_{p_{\mathrm{o}}}^{p_{\mathrm{w}}^{\mathrm{A}}} \frac{p}{\mu Z} \mathrm{~d} p=\frac{\left(p_{\mathrm{i}}\right)^{2}-\left(p_{\mathrm{w}}^{\mathrm{A}}\right)^{2}}{\bar{\mu} \bar{Z}}
\end{aligned}
$$

Because $Q_{\mathrm{sc}}=86.4 q_{\mathrm{sc}} L_{\mathrm{D}}$, the steady-state productivity equation for a horizontal well in a bottom-water-drive gas reservoir can be written in the form of pressure, pseudopressure, and square pressure:

$$
Q_{\mathrm{sc}}=\frac{542.87 \sqrt{k_{\mathrm{h}} k_{\mathrm{v}}} L T_{\mathrm{sc}}}{p_{\mathrm{sc}} T} \frac{\bar{p}\left(p_{\mathrm{i}}-p_{\mathrm{w}}^{\mathrm{A}}\right)}{\bar{\mu} \bar{Z} \frac{5}{6}\left(\ln \frac{4 H_{\mathrm{D}}}{\pi R_{\mathrm{wD}}}+\ln \tan \frac{\pi z_{\mathrm{wD}}}{2 H_{\mathrm{D}}}\right)}
$$

for $p \geq 21 \mathrm{MPa}$ (high-pressure) (42)

$$
Q_{\mathrm{sc}}=\frac{271.435 \sqrt{k_{\mathrm{h}} k_{\mathrm{v}}} L T_{\mathrm{sc}}}{p_{\mathrm{sc}} T} \frac{\left(m_{\mathrm{i}}-m_{\mathrm{w}}^{\mathrm{A}}\right)}{\frac{5}{6}\left\{\ln \frac{4 H_{\mathrm{D}}}{\pi R_{\mathrm{wD}}}+\ln \tan \frac{\pi z_{\mathrm{wD}}}{2 H_{\mathrm{D}}}\right\}}
$$

for $13 \mathrm{MPa}<p<21 \mathrm{MPa}$

$$
Q_{\mathrm{sc}}=\frac{271.435 \sqrt{k_{\mathrm{h}} k_{\mathrm{v}}} L T_{\mathrm{sc}}}{p_{\mathrm{sc}} T} \frac{\left(p_{\mathrm{i}}\right)^{2}-\left(p_{\mathrm{w}}^{\mathrm{A}}\right)^{2}}{\bar{\mu} Z \frac{5}{6}\left(\ln \frac{4 H_{\mathrm{D}}}{\pi R_{\mathrm{wD}}}+\ln \tan \frac{\pi z_{\mathrm{wD}}}{2 H_{\mathrm{D}}}\right)}
$$

for $p \leq 13 \mathrm{MPa}$ (low-pressure) (44)

\section{Applications}

Example 1 A horizontal well was located at the center of a bottom-water-drive gas reservoir. The reservoir properties and well data are listed in Table 1 . When the initial reservoir pressure and temperature were $14 \mathrm{MPa}$ and $95{ }^{\circ} \mathrm{C}$, the gas viscosity and $Z$-factor were $0.025 \mathrm{mPa} \cdot \mathrm{s}$ and 0.96 , respectively; when the initial reservoir pressure and temperature were $38 \mathrm{MPa}$ and $95{ }^{\circ} \mathrm{C}$, the gas viscosity and $Z$-factor were $0.035 \mathrm{mPa} \cdot \mathrm{s}$ and 1.053 , respectively.

Table 1 Reservoir and well data for Example 1

\begin{tabular}{cc}
\hline Parameter & Value \\
\hline Well radius $r_{\mathrm{w}}, \mathrm{m}$ & 360 \\
\hline Horizontal well length $L, \mathrm{~m}$ & 0.10 \\
The distance of the horizontal well to the lower boundary $z_{\mathrm{w}}, \mathrm{m}$ & 18 \\
\hline Horizontal permeability $k_{\mathrm{h}}, \mathrm{mD}$ & 0.15 \\
Vertical permeability $k_{\mathrm{v}}, \mathrm{mD}$ & 50 \\
Formation thickness $H, \mathrm{~m}$ & 0.5 \\
Reservoir temperature, ${ }^{\circ} \mathrm{C}$ & 20 \\
\hline
\end{tabular}


The productivity of the horizontal well was calculated from Eqs. (42)-(44) and the Canadian CMG simulation software, respectively. Figs. 2 and 3 show the curves calculated from Eqs. (42)- (44) proposed in this study are very close to the curves from the $\mathrm{CMG}$ simulation software, indicating that the new steady-state productivity equation for horizontal wells proposed in this paper has potential practical application.



Fig. 2 Horizontal well productivity calculated under $p_{\mathrm{i}}=14 \mathrm{MPa}$

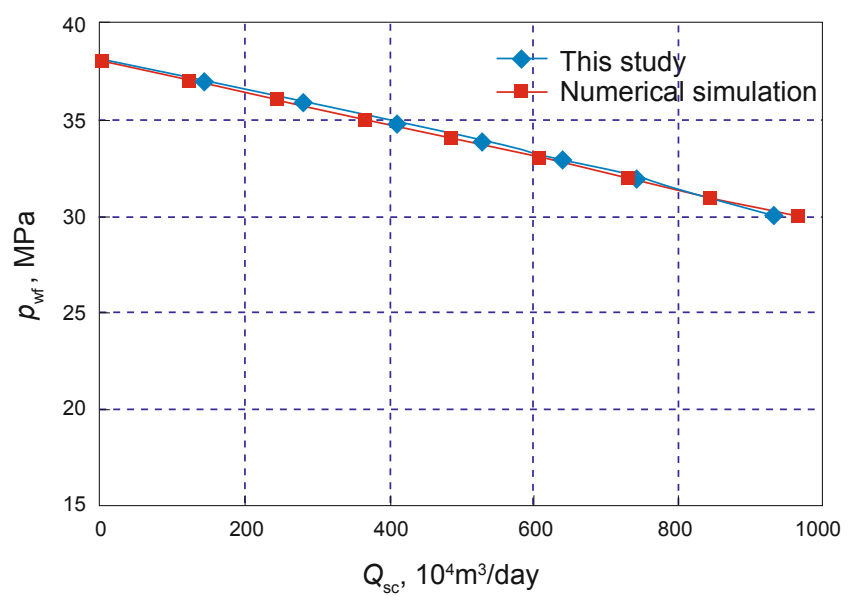

Fig. 3 Horizontal well productivity calculated under $p_{\mathrm{i}}=38 \mathrm{MPa}$

Example 2 A horizontal well was drilled in the Moxi reservoir, which located at Sichuan Province of southwest China.

The gas reservoir was a bottom-water-drive reservoir, with the initial reservoir pressure and temperature of 20.1 $\mathrm{MPa}$ and $80{ }^{\circ} \mathrm{C}$ respectively. The gas viscosity and $Z$-factor were $0.038 \mathrm{mPa} \cdot \mathrm{s}$ and 0.99 , respectively, under initial reservoir conditions. The pay thickness of the reservoir was about $4.2 \mathrm{~m}$. The horizontal section of the wellbore, nearly $3 \mathrm{~m}$ away from the lower boundary, was $516 \mathrm{~m}$ long, with a wellbore radius of $0.140 \mathrm{~m}$. Well test analysis indicates that the horizontal permeability in the reservoir was about $1 \mathrm{mD}$ while the vertical permeability approximately $0.1 \mathrm{mD}$. Table
2 is a summary of the actual well productivity and the well productivity calculated from the formulae proposed in this paper.

Table 2 A summary of the actual and calculated productivity of the horizontal well in the Moxi reservoir

\begin{tabular}{ccccc}
\hline $\begin{array}{c}\text { Date } \\
\text { mm/yyyy }\end{array}$ & $\begin{array}{c}\text { Shut-in wellhead } \\
\text { pressure, MPa }\end{array}$ & $\begin{array}{c}\text { Bottom hole } \\
\text { pressure, MPa }\end{array}$ & $\begin{array}{c}\text { Actual well } \\
\text { productivity } \\
10^{4} \mathrm{~m}^{3} / \text { day }\end{array}$ & $\begin{array}{c}\text { Calculated well } \\
\text { productivity } \\
10^{4} \mathrm{~m}^{3} / \text { day }\end{array}$ \\
\hline $12 / 2002$ & 20.1 & 20.1 & 0 & 0 \\
$1 / 2003$ & 20.1 & 19.1 & 9.4 & 10.3 \\
$3 / 2003$ & 18.5 & 17.5 & 9.2 & 8.6 \\
$5 / 2003$ & 15.5 & 14.3 & 7.2 & 8.5 \\
$7 / 2003$ & 14.4 & 13.2 & 7.1 & 7.9 \\
\hline
\end{tabular}

\section{Conclusions}

Using the idea of source and the Green function, the fundamental, 3D steady-state solution to the Laplace equation for horizontal wells in bottom-water-drive gas reservoirs was obtained, and then a novel productivity equation for horizontal wells producing at different pressures was derived. Two field case-studies were conducted and the comparisons between the results obtained from the new formula and the actual productivity and CMG simulation results show that the new equation can be used to predict the steady-state productivity of horizontal gas wells.

\section{Acknowledgements}

The authors are grateful for the financial support from the Open Fund (PLN1003) of State Key Laboratory of Oil and Gas Reservoir Geology and Exploitation (Southwest Petroleum University), and the National Science and Technology Major Project in the 11th Five-Year Plan (Grant No. 2008ZX05054).

\section{References}

Anklam E G and Wiggins M L. Horizontal-well productivity and wellbore pressure behavior incorporating wellbore hydraulics. Paper SPE 72361 presented at the SPE Production Operations Symposium, 16-19 April 2005, Oklahoma City, Oklahoma

Babu D K and Odeh A S. Productivity of a horizontal well. SPE Reservoir Engineering. 1989. 4(4): 417-421

Babu D K and Odeh A S. Productivity of a horizontal well: Appendices A and B. Paper SPE 18334 presented at the SPE Annual Technical Conference and Exhibition, 2-5 October 1988, Houston, Texas

Borisov J P. Oil production using horizontal and multiple deviation wells. The R \& D Translation Company, Bartlesville, Oklahoma, 1984

Billiter T, Lee J and Chase R. Dimensionless inflow-performancerelationship curve for unfractured horizontal gas wells. Paper SPE 72361 presented at the SPE Eastern Regional Meeting, 17-19 October 2001, Canton, Ohio

Caralaw H S and Jaeger J C. Conduction of heat in solids. Oxford University Press. 1946. 353-386.

Chen W, Zhu D and Hill A D. A comprehensive model of multilateral 
well deliverability. Paper SPE 64751 presented at the International Oil and Gas Conference and Exhibition in China, 7-10 November 2000, Beijing, China

Giger F M, Reiss L H and Jourdan A P. The reservoir engineering aspects of horizontal drilling. Paper SPE 13024 presented at the 59th Annual Technical Conference and Exhibition, 16-19 September 1984, Houston, Texas

Giger F M. Horizontal wells production techniques in heterogeneous reservoirs. Paper SPE 13710 presented at the Middle East Oil Technical Conference and Exhibition, 11-14 March 1985, Bahrain

Gradshteyn I S and Ryzhik I M. Table of Integrals, Series and Products (Seventh edition). Academic Press Inc., San Diego, CA., California, U.S.A, 46-49, 2007

Gringarten A C and Ramey H J. The use of source and Green's functions in solving unsteady-flow problems in reservoirs. SPE Jounral 1973. 5(13): 285-296 (Paper SPE 3818)

Helmy M W and Wattenbarger R A. Simplified productivity equations for horizontal wells producing at constant rate and constant pressure. Paper SPE 49090 presented at the SPE Annual Technical Conference and Exhibition, 27-30 September 1998, New Orleans, Louisiana

Joshi S D. Augmentation of well productivity using slant and horizontal wells. Journal of Petroleum Technology. 1988. 6(40): 729-739 (Paper SPE 15375)

Karcher B J and Giger F M. Some practical formulas to predict horizontal well behavior. Paper SPE 15430 presented at the SPE Annual Technical Conference and Exhibition, 5-8 October 1986, New Orleans, Louisiana

Kong X Y. Advanced Mechanics of Fluids in Porous Media. Hefei:
Science and Technology University Press. 1999: 103-125 (in Chinese)

Larsen L. Productivity computations for multilateral, branched and other generalized and extended well concepts. Paper SPE 36754 presented at the SPE Annual Technical Conference and Exhibition, 6-9 October 1996, Denver, Colorado

$\mathrm{Lu}$ J. An analytical solution of steady-state flow equation for the productivity of a horizontal well. Petroleum Exploration and Development. 1993. 20(Supplement): 135-140 (in Chinese)

$\mathrm{Lu}$ J. New productivity formulae of horizontal wells. Journal of Canadian Petroleum Technology. 2001. 40(10): 55-67

Lu J, Lin T, Rogers R, et al. A mathematical model of horizontal wells pressure drawdown and buildup. Journal of Canadian Petroleum Technology. 2002. 41(10): 45-57

$\mathrm{Lu}$ J, Lin T, Rogers R, et al. A supplemental discussion of productivity formulae of horizontal wells. Journal of Canadian Petroleum Technology. 2003. 42(10): 23-27

Merkulov V P. Le debit des puits devies et horizontaux. Neft. Khoz. 1958. 1(6): 51-56.

Reiss L H. Production from horizontal wells after five years. Journal of Petroleum Technology. 1987. 39(11): 1411-1416 (Paper SPE14338)

Renard G and Dupuy J M. Formation damage effects on horizontal-well flow efficiency. Journal of Petroleum Technology. 1991. 7(43): 786789 (Paper SPE 19414)

Zwillinger D. Standard Mathematical Tables and Formulae. New York: CRC Press. 1996. 103-104

(Edited by Sun Yanhua) 